\title{
Optically Controlled Ferroelectric Nanodomains for Logic-in-Memory Photonic Devices with Simplified Structures
}

\author{
Fei Xue, Xin He, Dharmaraj Periyanagounder, Zhigang Ji, Member, IEEE, Lain-Jong Li, \\ Jr-Hau He, Senior Member, IEEE, and Xixiang Zhang
}

\begin{abstract}
We report visible-light-induced ferroelectric nanodomain reversal in conductive van der Waals (vdW) ferroelectric $\alpha$-In2 $\operatorname{Se}_{3}$. We show its promising application in two-terminal optoelectronic memory devices. Compared to other vdW materials based optoelectronic memories, such a novel working prototype allows for the device operation confined within a single material channel bridging its two electrodes, which greatly reduces the complexities of device construction. In addition, using $\alpha$-In ${ }_{2} \mathrm{Se}_{3}$ memory devices we also demonstrate the universal OR and AND optical logic gates for logic-in-memory application. Our results provide a new avenue to design simplified structures of vdW materials based optoelectronic memories for dense device integration and next-generation computation.
\end{abstract}

Index Terms-In-memory computing, optoelectronic memories, van der Waals ferroelectrics

\section{INTRODUCTION}

$\mathrm{T}$ HE rapid developments of artificial intelligence and the Internet of Things have produced abundant data that needs to be massively processed and stored by multifunctional devices. Regarding optical data, optoelectronic memories with the functionalities of photon sensing and storing, play a pivotal role and gain considerable interests [1,2]. Although silicon-based optoelectronic memories have been commercialized for many years, the miniaturization of these silicon devices suffers from the limitation of physically separated photon capture and storage cells. Therefore, seeking

This paragraph of the first footnote will contain the date on which you submitted your paper for review. It will also contain support information, including sponsor and financial support acknowledgment.

The work was supported by KAUST with a funding ORS-2016-CRG5-2996 and City University of Hong Kong.

Fei Xue, Xin He, Dharmaraj Periyanagounder, Lain-Jong Li and Xixiang Zhang are with the Physical Science and Engineering Division, King Abdullah University of Science and Technology, Thuwal 23955-6900, Saudi Arabia (e-mail: fei.xue@kaust.edu.sa; xixiang.zhang@kaust.edu.sa).

Zhigang $\mathrm{Ji}$ is with National Key Laboratory of Science and Technology on Micro/Nano Fabrication, Shanghai Jiao Tong University, Shanghai, 200240, China.

$\mathrm{Jr}$-Hau He is with Department of Materials Science and Engineering, City University of Hong Kong, Kowloon, Hong Kong, China. a potential approach to combine these two functional cells together in a single device is particularly significant for high-density monolithic integration.

Fortunately, beneficial from the superior optical properties of several vdW materials including $\mathrm{MoS}_{2}$, their optoelectronic memories with fused functionalities of photon capture and storage have been successively demonstrated [3-13]. These devices are all tied to the regime of charge trapping and detrapping, which, according to the architectures, can be classified into two distinct groups. As shown in the top panel of Fig. 1a, a typical one (type 1) is that of transistor-like configuration $[7,9]$. In order to create charge trapping centers, the type 1 substrate surfaces normally need to be functionalized. Meanwhile, after optically writing into the photoactive channel, a gate pulse is essential for detrapping charges such that the stored optical information can be fully erased. Hence, the type 1 optoelectronic memories indeed require modified substrates and three terminals to accomplish writing and erasing operations, which hinders device integration particularly crossbar-structure construction. The other one (type 2) though has two terminals but is composed of a heterostructure with at least three dissimilar vdW materials $[12,13]$ as exhibited at the bottom panel of Fig. 1a. This heterostructure helps to the storage and retrieval of photo-generated charges. However, when fabricating a single device or device array, assembling of disparate vdW materials together seems a daunting task, which limits the practical use as well.

The charge trapping and detrapping mechanism inevitably complicates device architectures, which presents a challenge to integrate vdW-materials-based optoelectronic memories in a high density. To simplify and revolutionize the device structure a novel mechanism is highly anticipated. Here, we report a novel mechanism of optoelectronic memories at the device level whose operation only relates to the channel ferroelectric $\alpha-\operatorname{In}_{2} \mathrm{Se}_{3}$ with its two metal terminals. Upon light irradiation ferroelectric nanodomain reversal in $\alpha$ - $\operatorname{In}_{2} \mathrm{Se}_{3}$ channel is observed, which is distinct from the domain wall motions in our published paper [14]. The nanodomain flipping exhibits non-volatile properties and can be used to store light information. The endurance of optical writing and electrical erasing can be over 800 cycles. Using the as-fabricated $\alpha-\mathrm{In}_{2} \mathrm{Se}_{3}$ optoelectronic memories, we demonstrate the functionality of logic-in memory. 


\section{RESULTS AND DISCUSSION}

The $\alpha-\operatorname{In}_{2} \mathrm{Se}_{3}$ is a vdW ferroelectric crystal with switchable polarization down to the monolayer limit, which has gained considerable interest since its stable polarization was theoretically predicted in 2017 [14-24]. Using Scotch tape method, it can be easily exfoliated to ultrathin flakes. To fabricate $\alpha-\operatorname{In}_{2} \mathrm{Se}_{3}$ optoelectronic memories these cleaved $\alpha-\mathrm{In}_{2} \mathrm{Se}_{3}$ fakes were first transferred onto silicon substrates with a 300-nm oxide layer. Afterwards, a two-terminal device was formed by depositing two metal stripes (70-nm Au/5-nm Ti). In our $\alpha-\operatorname{In}_{2} \mathrm{Se}_{3}$ devices (Fig. 1b) the optical writing and electrical erasing processes are only associated with a ferroelectric channel with its two electrodes, which features a simplified architecture compared to other vdW materials-based devices (Fig. 1a). Raman spectrum was employed to identify the channel material (Fig. 1d). There are five typical Raman
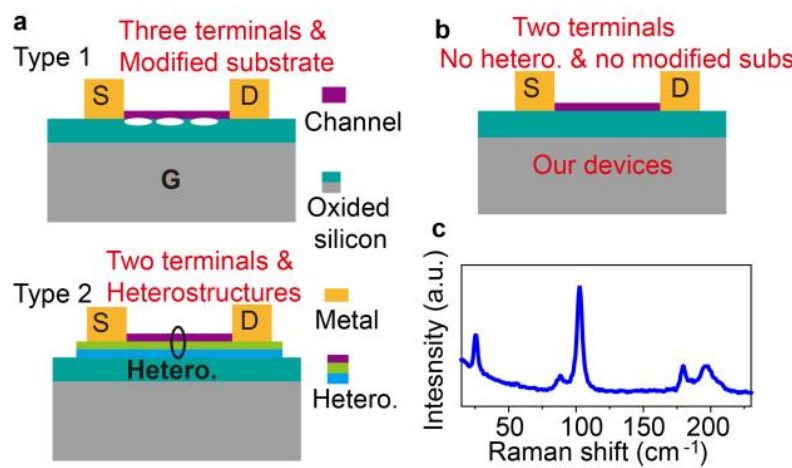

Fig. 1. (a) Conventional two types of optoelectronic memories based on two-dimensional materials. The red indicates the elements that are involved in device operations. (b) Our demonstrated device structure with only two terminals connecting a single ferroelectric flake. (c) Typical Raman spectrum of our used $\alpha-\ln _{2} \mathrm{Se}_{3}$ samples.

peaks, located at $27 / \mathrm{cm}, 89 / \mathrm{cm}, 104 / \mathrm{cm}, 180 / \mathrm{cm}$ and $195 / \mathrm{cm}$ respectively. These peaks, particularly the one at $89 / \mathrm{cm}$, assign our used $\alpha-\mathrm{In}_{2} \mathrm{Se}_{3}$ to hexagonal structure, which is distinguishable from rhombohedral counterpart [24]. As previously reported [14], the hexagonal $\alpha-\mathrm{In}_{2} \mathrm{Se}_{3}$ has a direct bandgap of $\sim 1.45 \mathrm{eV}$, very suitable for photoactive materials and optical devices with visible light sensing $[25,26]$.

Then we will look at ferroelectric polarization evolution with respect to light irradiation. Note that a halogen light was guided through fiber to illuminate the channel. A representative $\alpha-\mathrm{In}_{2} \mathrm{Se}_{3}$ device with channel width of $4.5 \mu \mathrm{m}$ and channel length of $1 \mu \mathrm{m}$ was taken as an example wherein the sample height is about $30-40 \mathrm{~nm}$ (Fig. 2a). To clearly visualize the polarization change, a small area (dashed square in Fig. 2a) of the $\alpha-\mathrm{In}_{2} \mathrm{Se}_{3}$ channel was selected and scanned using piezoresponse force microscope (PFM) as shown in Fig. 2b. The blue dashed curve indicates a ferroelectric domain wall across $\alpha$ - $\mathrm{In}_{2} \mathrm{Se}_{3}$ channel. After the application of a $-5 \mathrm{~V}$ poling pulse at T2 with $\mathrm{T} 1$ grounded, a PFM phase contrast occurs over the domain wall (the left-top panel of Fig. 2c) and the ferroelectric polarization will be aligned toward T2 like the schematic in the left panel of Fig. 2e. Due to six-fold in-plane structural asymmetry in $\alpha-\operatorname{In}_{2} \mathrm{Se}_{3}$ [27], the dipole $P$ presents $120^{\circ}$ rotation in the vicinity of domain wall. When the channel is poled by $-5 \mathrm{~V}$ and subsequently illuminated by light for $5 \mathrm{~s}$,
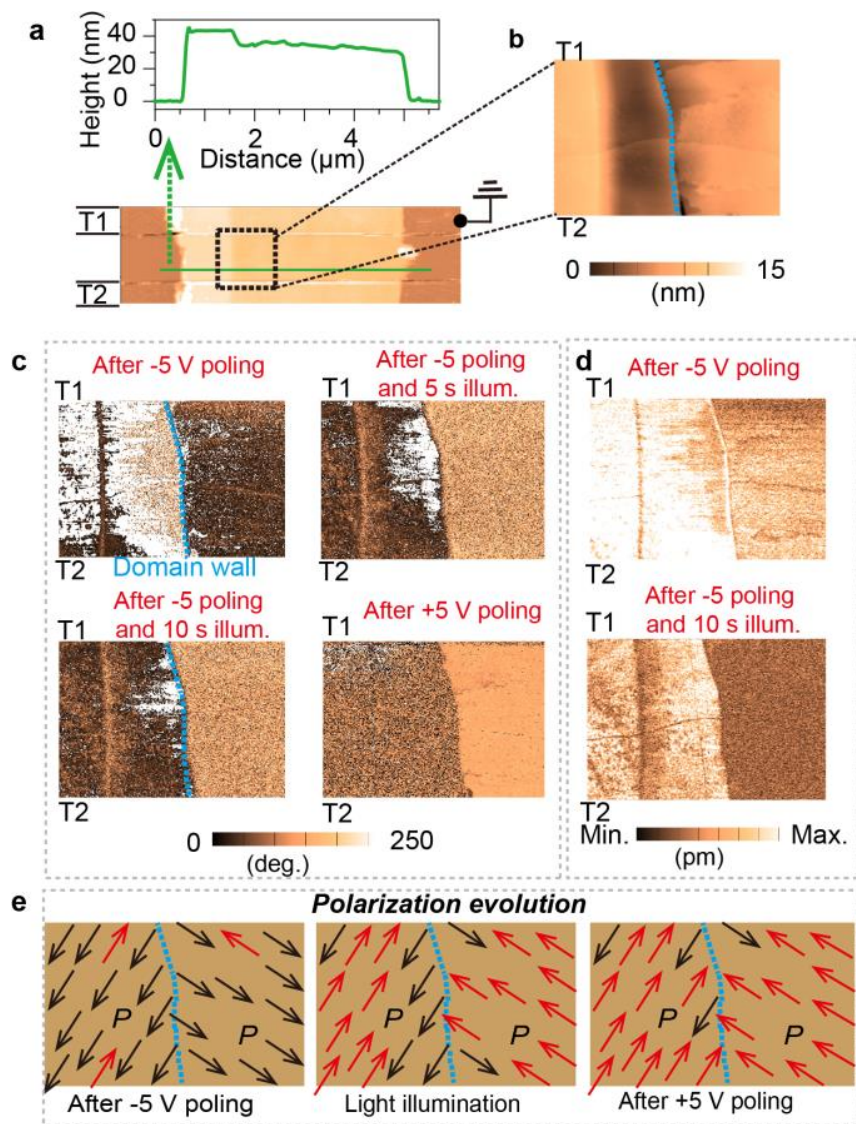

Fig. 2. (a) AFM image of a typical $\alpha-\ln _{2} \mathrm{Se}_{3}$ device. The channel height as marked by the green is shown at the top panel. (b) Enlarged channel topography of the dashed square area in (a). (c) PFM phase mapping and (d) PFM amplitude mapping with different poling conditions. (e) Illustrations of ferroelectric polarization switching under different conditions.

the related polarization at both sides of domain wall (the right-top panel of Fig. 2c) exhibits dramatic reversal. Upon light illumination, the domain wall that is parallel with the channel almost shows no change, in contrast with the reported optical-controlled domain wall motion [14]. As the illumination time is raised to $10 \mathrm{~s}$, the white and bright area is further reduced (left-bottom panel of Fig. 2c), suggesting that long irradiation time leads to more polarization switched toward $\mathrm{T} 2$. The resulting polarization is reminiscent of domain configuration with $+5 \mathrm{~V}$ poling (right-bottom panel of Fig. 2c, and the schematics in Fig. 2e). Notably, by scrutinizing the set of PFM mapping, it is observed that the switching dynamic is governed by the expansion of nanodomain: for example, the nanodomains residing at the left-bottom of PFM mapping initiate the switching, and then propagate. This process is consistent with the description in Kolmogorov-Avrami-Ishibashi (KAI) model. Additionally, PFM amplitude mapping before and after 10-s light illumination is also shown in Fig. $2 d$ to further validate optical-induced polarization switching in $\alpha$ - $\operatorname{In}_{2} \mathrm{Se}_{3}$. By contrast, upon $+5 \mathrm{~V}$ poling and subsequent light illumination for $10 \mathrm{~s}$, the polarization shows little change. Overall, the PFM measurement demonstrates optical control over $\alpha$ - $\operatorname{In}_{2} \mathrm{Se}_{3}$ ferroelectric polarization particularly when the channel domain is flipped by $-5 \mathrm{~V}$. Such an optical-induced polarization 

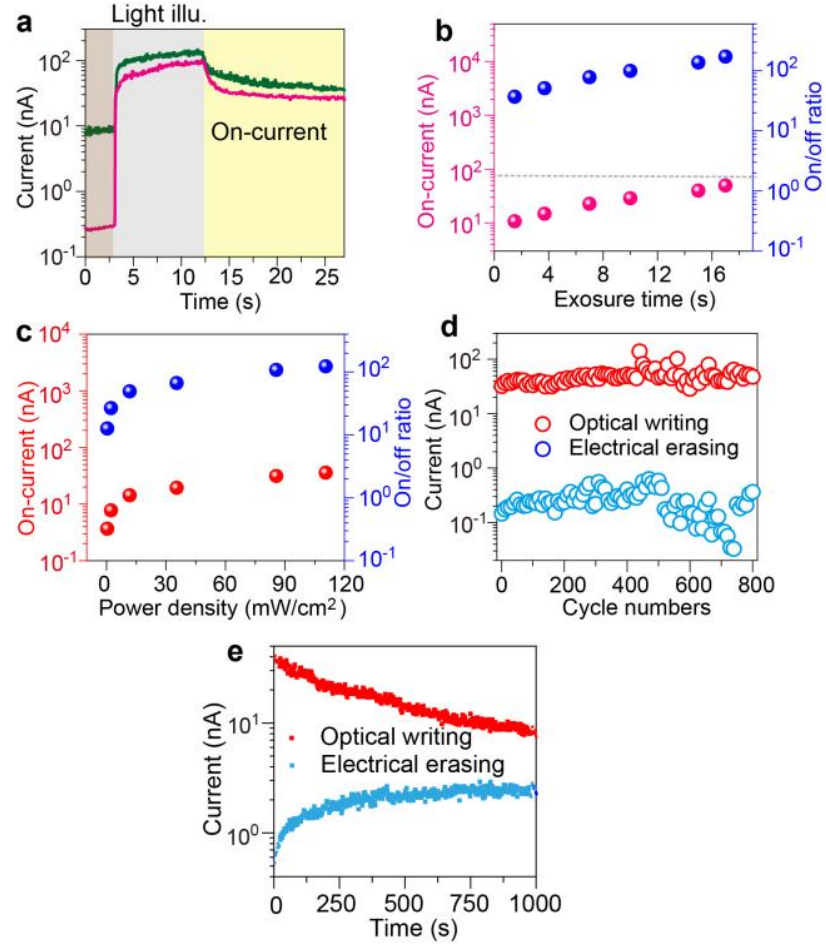

Fig. 3. (a) Time resolved non-volatile photoresponse current (on-current) under different polar ordering enforced either by $+5 \mathrm{~V}$ poling for the green or by $-5 \mathrm{~V}$ poling for the pink. The on-current is highlighted by the yellow background, while the dynamic photoresponse current is marked by the grey. (b) The on-current as a function of light exposure time after the application of $-5 \mathrm{~V}$ poling. (c) Power density dependent on-current with fixed $10 \mathrm{~s}$ light irradiation on the device. The polar ordering across the device channel is always set by $-5 \mathrm{~V}$ poling before each measurement. The corresponding on/off ratios are also plotted in right longitudinal coordinates as shown by the blue in $b$ and c. (d) and (e) Endurance and retention properties for the $a$ $-\mathrm{In}_{2} \mathrm{Se}_{3}$ device. Read bias in a-e: +0.3 V.
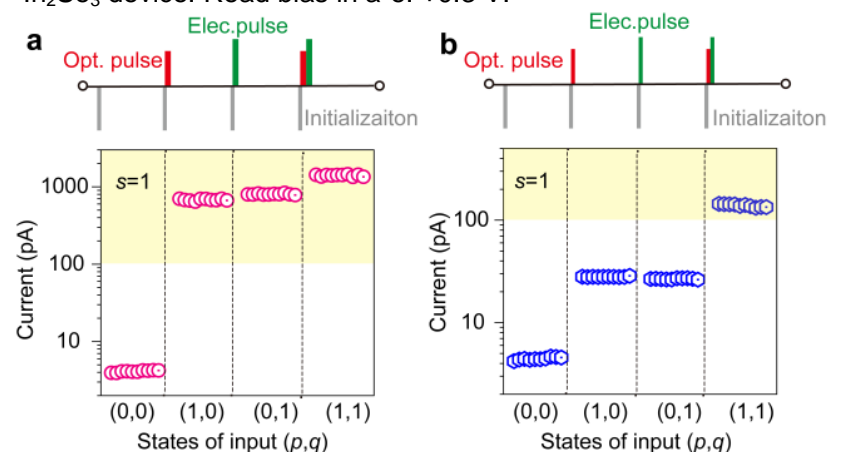

Fig. 4. Demonstration of universal (a) OR and (b) AND optical logic gates using another $\alpha-\operatorname{In}_{2} \mathrm{Se}_{3}$ optoelectronic memories. For the OR gate, optical pulses (red histograms at the top of panel a) with width of $10 \mathrm{~s}$ and power density of $85.6 \mathrm{~mW} / \mathrm{cm}^{2}$ represent " $p=1$ "; electrical pulses (green histograms at the top of panel a) with width of $3 s$ and amplitude of $+5 \mathrm{~V}$ indicate " $q=1$ "; the gate outputs of $(p, q)$ are marked by " $s$ ". For the AND gate, both the widths of optical and electrical pulses were reduced to $1 \mathrm{~s}$ and $0.8 \mathrm{~s}$, respectively. Note that the current magnitude above $100 \mathrm{pA}$ was designated to $s=1$.

reversal coincides with the observations in oxide-based ferroelectrics [28-31].

The charge trapping effect under light illumination has been ruled out for $\alpha$ - $\operatorname{In}_{2} \mathrm{Se}_{3}$ optoelectronic devices [14] The origin of our observations in this work (i.e., optically controlled nanodomain reversal) can be ascribed to the unbalanced screening effect on ferroelectric bound charges at the nanodomain walls. The nanodomain wall across $\alpha$ - $\operatorname{In}_{2} \mathrm{Se}_{3}$ channel is unusually stabilized by absorbed compensation charges, which, however, are prone to be disturbed by photogenerated carriers. The interaction between ferroelectric bound charges, compensation charges and photogenerated carriers results in $\alpha$ - $\operatorname{In}_{2} \mathrm{Se}_{3}$ ferroelectric nanodomain switching. We note that the in-depth understanding of the competition for these three factors needs future investigation. To phenomenologically give insight into the polarization evolution, we show its schematics with different poling conditions in Fig. $2 \mathrm{~d}$.

Having observed that visible light can induce ferroelectric nanodomain reversal in $\alpha$ - $\operatorname{In}_{2} \mathrm{Se}_{3}$, we investigated whether this effect could be employed to store and retrieve optical data. As revealed in Fig. $3 a$, the $+5 \mathrm{~V}$ and $-5 \mathrm{~V}$ electrical poling not only toggles the ferroelectric polarization, but also switch the current to a high value and a low value respectively. While the light is suddenly applied, the photoelectric effect happens across the $\alpha-\operatorname{In}_{2} \mathrm{Se}_{3}$ channel and the corresponding current dramatically increases. At the same time a large volume of photogenerated carriers can disturb the screening of compensation charges on polarization charges congregated at nanodomain walls, resulting in ferroelectric polarization flipping. Once the light irradiation is terminated, the currents are abruptly decreased but can still keep greater values (on-current; Fig. 3a), which are a signature (i.e., non-volatile photoresponses) of optoelectronic memories. Note that there are a large on/off ratio for $-5 \mathrm{~V}$ poling and a small value for $+5 \mathrm{~V}$ poling which are consistent with PFM results in Fig. 2. We note that although the non-volatile photoresponse has also been observed in oxide ferroelectrics [32,33], its origin is found to be associated with the charges trapping or vancancy formation, and comopletely different from what we have demonstrated here. Then we explored the effect of light exposure time and light intensity on the on-current upon $-5 \mathrm{~V}$ poling. As shown in Fig. $3 \mathrm{~b}$, it is observed that as the expose time increases the on-current and related on/off ratio will increase as well. However, there are saturation trends above certain power densities of light, which arises from the fully reversal of polarization. Moreover, the power dependent on-current and on/off ratio (Fig. 3c) also show such a behavior: first increases and then saturates. Endurance is important parameters to evaluate optoelectronic memories. We demonstrated that the optical writing and electrical erasing can be alternately performed for over 800 cycles (Fig. 3d). The retention property is also shown in Fig. 3e.

Finally, we use $\alpha$ - $\operatorname{In}_{2} \mathrm{Se}_{3}$ optoelectronic memories to demonstrate prospective applications. To achieve optical logic gates, the logic variables-optical pulse, electrical pulse, and output current-are defined as $p, q$ and $s$ respectively. For the OR gate, the optical pulses with a duration of $10 \mathrm{~s}$ and power density of $85.6 \mathrm{~mW} / \mathrm{cm}^{2}$ are designated as " $p=1$ " like the red histogram shown at the top of Fig. 4a whereas the electrical pulses having a 3-s duration and $+5 \mathrm{~V}$ amplitude seen as " $q=1$ ". For the AND gate, the widths of both optical and electrical pulses are decreased to $1 \mathrm{~s}$ and $0.8 \mathrm{~s}$ respectively. Such definitions are consistent with the published work [34,35]. When there exist either an optical or electrical signal, the corresponding variable can be considered " 1 "; otherwise, $p$ and $q$ are assigned to " 0 ". As the output current is over $100 \mathrm{pA}$, the 
variable $s$ can be deemed as " 1 "; otherwise $s=0$ Before every operation, the $\alpha$ - $\operatorname{In}_{2} \mathrm{Se}_{3}$ device was always electrically initialized to a low current state. Based on these normal definitions, we successfully demonstrate the universal OR and AND optical logic gates as shown in Fig. 4a and 4b.

\section{CONCLUSION}

We have demonstrated a novel device prototype of optoelectronic memories for in-memory computing, in which the ferroelectric nanodomain reversal can be used to write optical data. Compared to other vdW materials based optoelectronic memories [6-9,12,13], this type of device features simplified device structure whose optical writing and electrical erasing operation are just related to $\alpha$ - $\mathrm{In}_{2} \mathrm{Se}_{3}$ channel and its connected metal electrodes. Universal Boolean logic gates are implemented in this $\alpha-\operatorname{In}_{2} \mathrm{Se}_{3}$ device. Our results suggest that vdW materials based optoelectronic memories can be constructed with a simpler device architecture, which is paramount for realizing future hardware of image recognition and neuromorphic vison sensors.

\section{REFERENCES}

[1] E. R. Fossum, "CMOS image sensors: Electronic camera-on-a-chip," (in English), IEEE Transactions on Electron Devices, vol. 44, no. 10, pp. 1689-1698, Oct 1997.

[2] A. Shacham, K. Bergman, and L. P. Carloni, "Photonic Networks-on-Chip for Future Generations of Chip Multiprocessors," IEEE Transactions on Computers, vol. 57, no. 9, pp. 1246-1260, 2008.

[3] Z. G. Cheng, C. Rios, W. H. P. Pernice, C. D. Wright, and H. Bhaskaran, "On-chip photonic synapse," (in English), Science Advances, vol. 3, no. 9, Sep 2017.

[4] S. Jang, E. Hwang, Y. Lee, S. Lee, and J. H. Cho, "Multifunctional graphene optoelectronic devices capable of detecting and storing photonic signals," Nano Lett, vol. 15, no. 4, pp. 2542-7, Apr 82015.

[5] W. Jin $\mathrm{Hu}, \mathrm{Z}$. Wang, W. Yu, and T. Wu, "Optically controlled electroresistance and electrically controlled photovoltage in ferroelectric tunnel junctions," Nat Commun, vol. 7, p. 10808, Feb 292016.

[6] D. Lee et al., "Multibit $\mathrm{MoS}_{2}$ Photoelectronic Memory with Ultrahigh Sensitivity," Adv Mater, vol. 28, no. 41, pp. 9196-9202, Nov 2016.

[7] J. Lee et al., "Monolayer optical memory cells based on artificial trap-mediated charge storage and release," Nat Commun, vol. 8, p. 14734, Mar 242017.

[8] S. Lei et al., "Optoelectronic memory using two-dimensional materials," Nano Lett, vol. 15, no. 1, pp. 259-65, Jan 142015.

[9] K. Roy et al., "Graphene- $\mathrm{MoS}_{2}$ hybrid structures for multifunctional photoresponsive memory devices," Nat Nanotechnol, vol. 8, no. 11, pp. 826-30, Nov 2013.

[10] F. Zhou, J. Chen, X. Tao, X. Wang, and Y. Chai, "2D Materials Based Optoelectronic Memory: Convergence of Electronic Memory and Optical Sensor," Research, vol. 2019, pp. 1-17, 2019.
[11] F. Zhou et al., "Optoelectronic resistive random access memory for neuromorphic vision sensors," Nat Nanotechnol, vol. 14, no. 8, pp. 776-782, Aug 2019.

[12] M. D. Tran et al., "Two-Terminal Multibit Optical Memory via van der Waals Heterostructure," Adv Mater, vol. 31, no. 7, p. e1807075, Feb 2019.

[13] D. Xiang et al., "Two-dimensional multibit optoelectronic memory with broadband spectrum distinction," (in English), Nature Communications, vol. 9, Jul 272018.

[14] F. Xue et al., "Optoelectronic Ferroelectric Domain-Wall Memories Made from a Single Van Der Waals Ferroelectric," (in English), Advanced Functional Materials, Sep 202020.

[15] C. Cui, F. Xue, W.-J. Hu, and L.-J. Li, "Two-dimensional materials with piezoelectric and ferroelectric functionalities," npj 2D Materials and Applications, vol. 2, no. $1,2018$.

[16] W. Ding et al., "Prediction of intrinsic two-dimensional ferroelectrics in $\mathrm{In}_{2} \mathrm{Se}_{3}$ and other $\mathrm{III}_{2}-\mathrm{VI}_{3}$ van der Waals materials," Nat Commun., vol. 8, p. 14956, Apr 72017.

[17] C. Cui et al., "Intercorrelated In-Plane and Out-of-Plane Ferroelectricity in Ultrathin Two-Dimensional Layered Semiconductor $\mathrm{In}_{2} \mathrm{Se}_{3}$," Nano Lett., vol. 18, no. 2, pp. 1253-1258, Feb 142018.

[18] S. Wan, Y. Li, W. Li, X. Mao, W. Zhu, and H. Zeng, "Room-temperature ferroelectricity and a switchable diode effect in two-dimensional alpha-In2Se3 thin layers," Nanoscale, vol. 10, no. 31, pp. 14885-14892, Aug 92018.

[19] S. Y. Wan et al., "Nonvolatile Ferroelectric Memory Effect in Ultrathin -In2Se3," (in English), Advanced Functional Materials, vol. 29, no. 20, May 162019.

[20] F. Xue et al., "Gate-Tunable and Multidirection Switchable Memristive Phenomena in a Van Der Waals Ferroelectric," Adv. Mater., p. e1901300, May 302019.

[21] C. X. Zheng et al., "Room temperature in-plane ferroelectricity in van der Waals In2Se3," (in English), Science Advances, vol. 4, no. 7, Jul 2018.

[22] J. Xiao et al., "Intrinsic Two-Dimensional Ferroelectricity with Dipole Locking," Phys. Rev. Lett., vol. 120, no. 22, p. 227601, Jun 12018.

[23] Y. Zhou et al., "Out-of-Plane Piezoelectricity and Ferroelectricity in Layered alpha-In2Se3 Nanoflakes," Nano Lett., vol. 17, no. 9, pp. 5508-5513, Sep 132017.

[24] F. Xue et al., "Room-Temperature Ferroelectricity in Hexagonally Layered $\alpha-\operatorname{In}_{2} \mathrm{Se}_{3}$ Nanoflakes down to the Monolayer Limit," Advanced Functional Materials, p. 1803738, 2018.

[25] R. B. Jacobs-Gedrim et al., "Extraordinary Photoresponse in Two-Dimensional In2Se3 Nanosheets," (in English), Acs Nano, vol. 8, no. 1, pp. 514-521, Jan 2014.

[26] J. O. Island, S. I. Blanter, M. Buscema, H. S. van der Zant, and A. Castellanos-Gomez, "Gate Controlled Photocurrent Generation Mechanisms in High-Gain In(2)Se(3) Phototransistors," Nano Lett, vol. 15, no. 12, pp. 7853-8, Dec 92015.

[27] F. Xue et al., "Multidirection Piezoelectricity in Monoand Multilayered Hexagonal alpha-In2Se3," ACS Nano, vol. 12, no. 5, pp. 4976-4983, May 222018. 
[28] Y. D. Liou et al., "Deterministic optical control of room temperature multiferroicity in $\mathrm{BiFeO} 3$ thin films," Nat Mater, vol. 18, no. 6, pp. 580-587, Jun 2019.

[29] F. Rubio-Marcos et al., "Reversible optical control of macroscopic polarization in ferroelectrics," Nature Photonics, vol. 12, no. 1, pp. 29-32, 2017.

[30] G. Vats, Y. Bai, D. Zhang, J. Juuti, and J. Seidel, "Optical Control of Ferroelectric Domains: Nanoscale Insight into Macroscopic Observations," Advanced Optical Materials, vol. 7, no. 11, p. 1800858, 2019.

[31] M. M. Yang and M. Alexe, "Light-Induced Reversible Control of Ferroelectric Polarization in $\mathrm{BiFeO}_{3}, " \mathrm{Adv}$ Mater, vol. 30, no. 14, p. e1704908, Apr 2018.

[32] W. J. Hu et al., "Colossal X-Ray-Induced Persistent Photoconductivity in Current-Perpendicular-to-Plane Ferroelectric/Semiconductor Junctions," (in English), Advanced Functional Materials, vol. 28, no. 6, Feb 7 2018.

[33] A. Bhatnagar, Y. H. Kim, D. Hesse, and M. Alexe, "Persistent Photoconductivity in Strained Epitaxial BiFeO3 Thin Films," (in English), Nano Letters, vol. 14, no. 9, pp. 5224-5228, Sep 2014.

[34] F. C. Zhou, Y. H. Liu, X. P. Shen, M. Y. Wang, F. Yuan, and Y. Chai, "Low-Voltage, Optoelectronic $\mathrm{CH} 3 \mathrm{NH} 3 \mathrm{PbI} 3-\mathrm{xClx}$ Memory with Integrated Sensing and Logic Operations," (in English), Advanced Functional Materials, vol. 28, no. 15, Apr 112018.

[35] F. C. Zhou and Y. Chai, "Near-sensor and in-sensor computing," (in English), Nature Electronics, vol. 3, no. 11, pp. 664-671, Nov 2020. 